\title{
RÉGIMEN LEGAL-AMBIENTAL APLICABLE A PROYECTOS INMOBILIARIOS
}

\author{
Francisco Javier González Silva \\ Profesor de Derecho Ambiental \\ Universidad Católica del Norte \\ Sede Coquimbo
}

La dictación de una nueva Ley trae usualmente como consecuencia el que se hagan innumerables interpretaciones acerca de su verdadero sentido y alcance.

En materia legal ambiental, este fenómeno se ha observado con especial significancia, pues, en muchos aspectos, la nueva Ley de Bases del Medio Ambiente y sus Reglamentos complementarios, no son del todo claros.

Uno de los tópicos que ha sido objeto de gran cantidad de interpretaciones está referido al cómo determinar cuál es el estatuto legal ambiental aplicable los proyectos inmobiliarios, específicamente, en lo relativo a la obligación de estos de ingresar al Sistema de Evaluación de Impacto Ambiental.

Lo anterior, en la actualidad, reviste una especial importancia, pues, en el mes de septiembre recién pasado existían 38 proyectos inmobiliarios iniciados con anterioridad al 02 de septiembre de ese año - momento en el cual entró en vigencia el D.S. 131, modificatorio del S.E.I.A. - de los cuales, 11 de ellos, no se sometieron al S.E.I.A. Por esta razón, esos proyectos no han podido hasta la fecha obtener de parte de las municipalidades respectivas la Recepción Definitivas de sus obras.

Ciertamente, una correcta labor interpretativa, debe tratar de distinguir claramente cuales son los distintos y posibles estatutos legales aplicables a los proyectos Inmobiliarios.

En este sentido, como primer paso, es necesario señalar los potenciales estatutos legales ambientales aplicables, en los siguientes términos :

- Estatuto Legal - Ambiental Ley 19.300 : Ley de Bases del Medio Ambiente (D.0. 09/03/95)

- Estatuto Legal - Ambiental D.S. 30: Reglamento del S.E.I.A. (D.O. $03 / 04 / 97)$

- Estatuto Legal - Ambiental D.S. 131: Modificación del S.E.I.A. (D.O. $02 / 09 / 98)$

El primero de ellos, esto es, el Estatuto Legal - Ambiental Ley 19.300, de Bases del Medio Ambiente (D.O. 09/03/95) es aplicable a todos los proyectos inmobiliarios ejecutados entre el 09 de Marzo de 1995 y el 03 de Abril de 1997, es decir, entre el 
momento en que entró en vigencia la Ley de Bases del Medio Ambiente y el momento en el cual entró en vigencia el Reglamento al S.E.I.A.

Según este estatuto jurídico, específicamente lo señalado en el artículo 10 letra h) de la L.B.M.A. deben Ingresar al S.E.I.A. todos aquellos proyectos inmobiliarios que modifiquen planes regionales de desarrollo urbano, planes intercomunales, planes reguladores comunales y planes seccionales, además de, todos aquellos ubicados en zonas declaradas latentes o saturadas.

Sin embargo, no obstante que este estatuto establece claramente qué proyectos inmobiliarios deben ingresar al S.E.I.A., presenta el gran defecto de no definir un aspecto básico, cual es, que es lo que se entenderá por proyecto inmobiliario.

En este sentido, nuestra opinión es que no puede exigirse el ingreso al S.E.I.A. a proyectos respecto de los cuales no existen criterios legales, ni generales ni particulares, para calificarlos como proyectos inmobiliarios.

Por otra parte e invocando el artículo primero transitorio de la ley 19.300 , - que establece que el S.E.I.A. regulado por ella entrará en vigencia una vez publicado en el Diario Oficial el Reglamento que lo regule -, llegamos a la misma conclusión de que estos proyectos no son alcanzados por la obligación de entrar al S.E.I.A.

En consecuencia, no cabe exigir el ingreso al S.E.I.A. a proyectos inmobiliarios iniciados en forma previa a la entrada en vigencia del Reglamento al S.E.I.A. respectivo, hecho ocurrido el 03 de marzo de 1997.

Importante es señalar que esta conclusión interpretativa es coincidente con la de Conama, la cual, dio una solución integral a este problema dictando una minuta interpretativa aplicable a la etapa de transición del S.E.I.A. que va desde la entrada en vigencia la de la Ley 19.300 (09/03/95) y la entrada en vigencia del Reglamento al S.E.I.A. (03/04/97).

En esa minuta, se señala expresamente que si un proyecto o actividad se encontraba en ejecución al momento de entrar en vigencia el Reglamento, el S.E.I.A. no se le aplica y, por lo tanto, ese proyecto está exento de someterse a éste, toda vez que tal situación no se encuadra en la descripción de los supuestos exigidos por los artículos 8 y 9 de la Ley 19.300

Para efecto de lo anterior, debe entenderse por etapa de ejecución de un proyecto la realización de obras, acciones o medidas tendientes a materializar una o más de las siguientes etapas: levantamiento de información en terreno, señalando las acciones y obras necesarias para la recolección de datos, en caso de ser procedente, construcción, operación y cierre y/o abandono.

El segundo Estatuto Legal - Ambiental, esto es, el D.S. 30 Reglamento al S.E.I.A. (D.O. 03/04/97) es aplicable a todos los proyectos inmobiliarios ejecutados entre el 03 de Abril de 1997 y el 02 de Septiembre de 1998, es decir, entre el momento en que entró en vigencia el Reglamento al S.E.I.A. y el momento en el cual entró en vigencia la modificación introducida a la letra h) del artículo 3 del Reglamento al S.E.I.A., la cual está referida, entre otras materias, a los proyectos inmobiliarios.

Según este estatuto jurídico, específicamente lo señalado en el artículo 3 letra h) del Reglamento al S.E.I.A. deben Ingresar al S.E.I.A. todos aquellos proyectos inmobi- 
liarios que modifiquen planes regionales de desarrollo urbano, planes intercomunales, planes reguladores comunales y planes seccionales, además de, todos aquellos ubicados en zonas declaradas latentes o saturadas.

Sin embargo, no obstante que a través de este segundo estatuto, se establece nuevamente $y$ en forma clara el qué proyectos inmobiliarios deben ingresar al S.E.I.A., otra vez nos encontramos con el gran defecto de que no se define el aspecto básico de saber qué es lo que se entenderá por proyecto inmobiliario.

En este sentido, nuestra posición sigue siendo postulando no puede exigirse el ingreso al S.E.I.A. a proyectos respecto de los cuales no existen criterios legales, ni generales ni particulares, para calificarlos de proyectos inmobiliarios, no obstante que el artículo primero transitorio de la Ley 19.300 estableció que el S.E.I.A. regulado en ella entraría en vigencia una vez publicado en el Diario Oficial el Reglamento respectivo.

Lo anterior es porque éste último Reglamento, tras su dictación, no estableció qué se entiende por proyecto inmobiliario, estableciendo sólo, y en términos genéricos, que estos deben ingresar al S.E.I.A.

Nuestra conclusión interpretativa es coincidente con la de Conama, la cual, si bien no ha dado una solución integral a este problema dictando una alguna minuta general interpretativa similar a la que se dictó para dar solución a los casos en los que se les aplica el primer estatuto ya mencionado, en la práctica, si ha hecho una muy importante distinción en relación con los proyectos inmobiliarios que ya comenzaron su etapa de ejecución y que no ingresaron al S.E.I.A.

Esta distinción es la siguiente:

A Aquellos proyectos en ejecución que NO cumplen las caracteristicas cuantitativas y cualitativas que según la modificación al Reglamento al S.E.I.A. (D.S. 131. $02 / 09 / 98$ ) son necesarios para estar en presencia de un proyecto Inmobiliario :

No se les exige entrar al Sistema de Impacto Ambiental.

Esta posición se basa en un criterio interpretativo de la Ley que sostiene que si estos proyectos no fueron alcanzados por la definición de proyecto inmobiliario introducida por el D.S. 30 , se reputa, entonces, que nunca lo fueron.

A Aquellos proyectos en ejecución que SI cumplen las características cuantitativas y cualitativas que según la modificación al Reglamento al S.E.I.A. (D.S. 131. 02/09/98) son necesarios para estar en presencia de un proyecto Inmobiliario :

Las distintas Coremas a lo largo del pais, que son autónomas en su funcionamiento, han dado diferentes soluciones al problema que se presenta cuando estos proyectos deciden ingresar al S.E.I.A.. Estas distintas soluciones han sido las siguientes:

- Rechazar los proyectos inmobiliarios ingresados al S.E.I.A. en su etapa de ejecución y no en forma previa.

El fundamento de esta postura está en el Principio Preventivo de nuestra legislación y que implicaría que éstos proyectos no cumplirían con la Legislación aplicable a los mismos, dentro de la cual, están las disposiciones legales que les obligan a evaluar previamente - y no durante su ejecución - el impacto ambiental. 
Esta posición que ha sido bastante fuerte, coloca ha estos proyectos en una dificil situación toda vez que, nuestra legislación no consagra un mecanismo de regularización de aquellos que se ejecuten sin el cumplimiento de las disposiciones legales ambientales.

- Declararse Incompetente para conocer los proyectos inmobiliarios ingresados al S.E.I.A. en su etapa de ejecución y no en forma previa.

El fundamento de esta postura está en los principios generales de Derecho Administrativo, en virtud de los cuales, los organismos públicos sólo pueden cumplir con las facultades y atribuciones que la propia Ley les asigna en forma expresa.

Así las cosas, algunas Coremas, basados en el principio preventivo y en lo establecido en el artículo 8 de la ley 19.300, han entendido que ellas sólo son competentes para aceptar ingresos al S.E.I.A. a proyectos nuevos que se presentan en forma previa a su ejecución_y no durante ella.

Esta posición que también ha sido bastante fuerte, coloca ha estos proyectos, como ya se señaló, en una difícil situación toda vez que nuestra legislación no consagra un mecanismo de regularización de los mismos y, lo que es peor, los deja en un "Limbo legal" imposible de solucionar, ajenos a toda posibilidad de obtener una certificación ambiental e, incluso, de obtener los permisos ambientales en forma sectorial, pues, los distintos servicios públicos, llamados a concederlos, no los entregan cuando se trata de aquellos permisos de carácter ambiental que deben obtenerse necesariamente al través del S.E.I.A.

Aceptar a tramitación al S.E.I.A. los proyectos inmobiliarios en ejecución o ya terminados.

Para dar solución al problema de aquellos proyectos inmobiliarios iniciados con anterioridad al 02 de septiembre de 1998 que no se sometieron al S.E.I.A, muchas empresas inmobiliarias o constructoras afectadas han sugerido a la Conama, en algunos casos, que instruya a las Direcciones de Obras Municipales a fin de que estas otorguen las recepciones definitivas de los proyectos en cuestión. En otros casos le han sugerido que oficie a las respectivas Coremas para que estas acepten a tramitación estos proyectos sugiriendo incluso que el ingreso al S.E.I.A. se produzca a través de una Declaración de Impacto Ambiental.

Este planteamiento ha sido analizado con la Conama, quienes han planteado que acceder a esta solución traería eventuales problemas de legalidad, puesto que, en una D.I.A. se declara bajo juramento el estar cumpliendo la Legislación Ambiental vigente, cosa que, en la práctica, no se produciría, toda vez que no se estaría evaluando el impacto ambiental en forma previa como lo exige el artículo 8 y 9 de la Ley. En ese sentido esta solución podría ser portadora de problemas de legalidad.

Por esta razón, y haciendo una labor interpretativa dentro de márgenes legales tolerables, nuestra posición postula que el único camino posible para dar solución a los casos pendientes de recepción municipal - relacionados con aquellos proyectos inmobiliarios en ejecución o ya concluidos al momento de la dictación del D.S. 131, sea que fueron alcanzados o no por la definición de proyecto inmobiliario que este instrumento legal consagra -, es la de presentar un consolidado, en el que se incluyan todos los casos que están en la misma situación, a consideración de la Dirección Ejecutiva de la Comi- 
Francisco Javier González Silva - Régimen Legal-Ambiental aplicable a Proyectos...

sión Nacional del Medio Ambiente, solicitando que sean expresa y taxativamente exceptuados de la obligación de entrar al S.E.I.A.

Este pronunciamiento formal podrá ser oponible ante las Direcciones de Obras de las Municipalidades las que deberán conceder las recepciones finales de las obras.

El fundamento de esta postura radica simplemente en que no puede exigirse el ingreso al S.E.I.A. a proyectos respecto de los cuales no existía al momento de su ejecución, criterios legales, ni generales ni particulares, para calificarlos como proyectos inmobiliarios de aquellos que deben evaluar previamente su impacto ambiental.

El tercer Estatuto Legal - Ambiental, esto es, el D.S. 131 modificatorio del Reglamento al S.E.I.A. (D.O. 02/09/98) es aplicable

es aplicable a todos los proyectos inmobiliarios ejecutados con posterioridad al 02 de Septiembre de 1998, momento en el cual, entró en vigencia la modificación introducida a la letra h) del artículo 3 del Reglamento al S.E.I.A., la cual está referida, entre otras materias, a los proyectos inmobiliarios.

Según este estatuto, deben ingresar al S.E.I.A. todos aquellos proyectos ubicados en zonas comprendidas en planes regionales de desarrollo urbano, planes intercomunales, planes reguladores comunales y planes seccionales....... y siempre y cuando los modifiquen o exista declaración de zona saturada o latente.

El gran avance logrado con esta última modificación es que por fin se define el aspecto básico de saber qué es lo que se entenderá por proyecto inmobiliario, existiendo, en consecuencia, un criterio legal para calificarlos como tales.

En efecto, señala expresamente este D.S. 131, que se entenderá por proyecto inmobiliario los siguientes:

Aquellos conjuntos que contemplen obras de edificación y urbanización cuyo destino sea habitacional $\mathrm{y} / \mathrm{o}$ equipamiento y que presenten alguna de las siguientes caracteristicas:

i) Que se emplacen en áreas urbanizables, de acuerdo al instrumento de planificación correspondiente, y requieran de sistemas propios de producción y distribución de agua potable y de recolección, tratamiento y disposición de aguas servidas ; o

i) Que den lugar a la incorporación al dominio nacional de uso público de vías expresas, troncales, colectoras o de servicios

En todo caso, se entenderán comprendidas en esta disposición aquellos conjuntos que se emplacen en una superficie de 7 o más hectáreas o consulten la construcción de 300 o más viviendas o edificios de uso público con una capacidad para 5.000 o más personas o con 1.000 o más estacionamientos

Nuestra posición postula que esta última modificación hecha al S.E.I.A. para los proyectos inmobiliarios a desarrollar con posterioridad al 02 de septiembre de 1998 (D.S. 131), es bastante clara y pensamos que, por lo tanto, no está sujeta a mayores interpretaciones. 